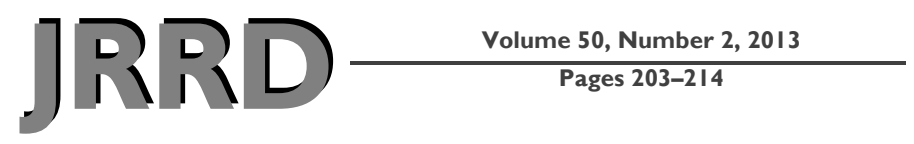

\title{
Comparison of changes in heart rate variability and sacral skin perfusion in response to postural changes in people with spinal cord injury
}

\author{
Yih-Kuen Jan, PT, PhD $;^{1-2^{*}}$ Mark Anderson, PT, PhD; ${ }^{1}$ Jeanine Soltani, PT, PhD; ${ }^{3}$ Stephanie Burns, PT, PhD; ${ }^{4}$ \\ Robert D. Foreman, $\mathbf{P h D}^{2}$ \\ Departments of ${ }^{1}$ Rehabilitation Sciences and ${ }^{2}$ Physiology, University of Oklahoma Health Sciences Center, Oklahoma \\ City, OK; ${ }^{3}$ Department of Inpatient Physical Medicine, Mercy Health Center, Oklahoma City, OK; ${ }^{4}$ Department of \\ Physical Therapy, Department of Veterans Affairs Medical Center, Oklahoma City, OK
}

\begin{abstract}
The current clinical practice has established guidelines to assess influences of severity of autonomic injury on the control of heart and blood pressure following spinal cord injury (SCI). However, the influences of SCI-induced autonomic impairment on microvascular dysfunction have not yet been established. Heart rate variability (HRV) has been shown to be a potential tool for quantifying residual sympathovagal regulation of the cardiovascular system following SCI and may be used to assess the effect of autonomic injury on skin microvascular dysfunction. A total of 26 people were recruited into the study, including 12 people with SCI and 14 nondisabled controls. HRV and sacral skin intervals and sacral skin perfusion were continually recorded during $10 \mathrm{~min}$ upright and $10 \mathrm{~min}$ prone postures. The sympathovagal balance was defined as the ratio of the power of the low frequency to the high frequency of HRV. The results showed that postural changes of nondisabled people produced significant changes in the sympathovagal balance; lower sympathovagal balance was associated with higher skin perfusion $(p<0.05)$. People with SCI did not show a significant change of HRV and skin perfusion in response to postural changes. In this study, we have demonstrated that the sympathovagal balance assessed by HRV was associated with the skin vasoconstrictive response to postural changes.
\end{abstract}

Key words: autonomic nervous system, blood flow oscillations, laser Doppler, heart rate, microvascular function, postural change, pressure ulcer, skin blood flow, spectral analysis, spinal cord injury.

\section{INTRODUCTION}

Following spinal cord injury (SCI), the deprivation of autonomic innervation of the cardiovascular system results in several clinical symptoms (e.g., autonomic dysreflexia, bradycardia, and orthostatic hypotension) and higher risk for cardiovascular diseases [1-2]. The current clinical practice (International Standards for the Neurological Classification of Spinal Cord Injury) focuses on the assessment of motor and sensory impairments following SCI but has not established a comprehensive assessment of autonomic impairments. Recently, the American

Abbreviations: ASIA = American Spinal Injury Association, $\mathrm{BFO}=$ blood flow oscillation, $\mathrm{C}=$ cervical, ECG = electrocardiography, HF = high frequency, HRV = heart rate variability, LDF = laser Doppler flowmetry, LF = low frequency, NS = not significant, pNN50 = percentage of adjacent cycles that are $>50 \mathrm{~ms}$ apart, rMSSD = root-mean-square successive differences in milliseconds, R-R = beat-to-beat interval of heart rate, SCI = spinal cord injury, SDNN = standard deviation of all normal-to-normal intervals, $\mathrm{T}=$ thoracic.

*Address all correspondence to Dr. Yih-Kuen Jan, PT, PhD; Rehabilitation Research Laboratory, Department of Kinesiology and Community Health, University of Illinois at Urbana-Champaign, 1206 S Forth St, MC-588, Champaign, IL 61820; 217-300-7253; fax: 217-333-2766.

Email: vian@illinois.edu

http://dx.doi.org/10.1682/JRRD.2011.08.0138 
Spinal Injury Association (ASIA) and the International Spinal Cord Society convened a task force to determine appropriate assessments to meet the clinical needs [3-4]. The task force has published an autonomic standards assessment to quantify autonomic dysfunction of the heart; blood pressure; sweating; temperature regulation; bronchopulmonary system; and urinary track, bowel, and sexual function. However, assessment of the effect of autonomic control on skin microcirculation has not been developed. This assessment is needed to evaluate the level and severity of damage to autonomic pathways and its influences on microvascular dysfunction-associated secondary complications (e.g., pressure ulcers) [2,4-5].

Skin blood flow oscillations (BFOs) contain five periodic components, including metabolic (0.0095$0.02 \mathrm{~Hz})$, neurogenic $(0.02-0.05 \mathrm{~Hz})$, myogenic $(0.05-$ $0.15 \mathrm{~Hz})$, respiratory $(0.15-0.4 \mathrm{~Hz})$, and cardiac $(0.4-$ $2.0 \mathrm{~Hz}$ ) origins [6-7]. The neurogenic control is related to the autonomic nervous system, including central cardiac and regional skin sympathetic innervation [8-10]. We demonstrated that the neurogenic control is independent of cutaneous axon reflexes in our previous studies [7,11], while the effect of SCI on the sympathetic outflow to the skin and the heart still needs to be studied [12-13]. Based on the ischemia theory of pressure ulcers [14], impaired microvascular function is associated with higher risk for pressure ulcers. In order to better understand the influences of SCI-induced sympathetic dysfunction on skin blood flow, the relationship between the autonomic impairment and microvascular dysfunction needs to be quantified. Among available tools to assess autonomic functions [15], heart rate variability (HRV) has shown promise as a way to quantify autonomic dysfunction on skin microcirculation.

HRV has shown great potential for quantifying residual autonomic functions of the cardiovascular system in people with SCI [2,16-19]. HRV is a periodic variation of beat-to-beat intervals of heart rate (R-R) (i.e., R-R intervals shown in the electrocardiogram where $\mathrm{R}$ represents early depolarization of the ventricles), with higher variations indicating lower risk for cardiovascular diseases [20]. The guideline for measurements and interpretations of HRV was developed by the Task Force of the European Society of Cardiology and the North American Society of Pacing and Electrophysiology [21]. Various indices have been developed to quantify HRV and can be classified as time and frequency domains. In the frequency domain, spectral analysis of HRV reveals two characteristic frequencies: one is defined as low frequency (LF) (ranged between $0.04 \mathrm{~Hz}$ and $0.15 \mathrm{~Hz}$ ) and the other is defined as high frequency (HF) (ranged between $0.15 \mathrm{~Hz}$ and $0.4 \mathrm{~Hz}$ ) [22]. HF of HRV is associated with parasympathetic outflow to the heart via the vagus nerve [21]. LF of HRV relates to activities of both sympathetic and parasympathetic systems, although research studies indicate that the LF of HRV may reflect the baroreflex rather than the sympathovagal activity [23]. The ratio of LF to HF has been widely used as an index of sympathovagal balance for assessing cardiovascular regulation. SCI not only causes autonomic impairments but also impairs baroreflex activity; thus, the use of LF to evaluate sympathovagal activity in people with SCI requires careful interpretation. Time domain parameters of HRV include the standard deviation of all normalto-normal intervals (SDNN), the percentage of adjacent cycles that are $>50 \mathrm{~ms}$ apart (pNN50), and the rootmean-square successive differences in milliseconds (rMSSD) [24]. SDNN reflects the sympathetic and parasympathetic influence on HRV [25], and pNN50 and rMSSD reflect vagal modulation of the sinoatrial node. Although HRV has shown promise as a tool to quantify residual autonomic functions in people with SCI, few studies have investigated the relationship between the changes of HRV and other physiological measurements following SCI [2]. In our case, we are particularly interested in understanding the influences of SCI-induced autonomic dysfunction on skin microcirculation and risk for ischemic injury and pressure ulcers [13].

To understand the influences of autonomic dysfunction on skin perfusion, we attempted to compare changes in the sympathovagal balance assessed by HRV and skin perfusion in response to postural changes in people with SCI. In this study, we hypothesized that nondisabled controls would have a significant change of the LF to HF ratio in response to postural changes and an increase of the LF to HF ratio is associated with a decrease in skin perfusion. We also hypothesized that people with SCI would not have a significant change of the LF to HF ratio and of skin perfusion in response to postural changes. The findings from this study will provide initial evidence to demonstrate a relationship between sympathovagal balance and skin perfusion. Through the use of this method, we intend to investigate the influences of various levels of autonomic damage on microvascular function in people with SCI. 


\section{METHODS}

\section{Subjects}

A total of 26 participants were enrolled in this study, including 12 people with SCI and 14 nondisabled controls. The inclusion criteria included traumatic SCI between the cervical (C)4 and thoracic (T)12 levels, time post-SCI at least 6 mo, use of a wheelchair as a primary means of mobility. The exclusion criteria included the presence of diabetes, pressure ulcers, or cardiopulmonary diseases or use of any medications that might affect cardiopulmonary function. The demographic data of participants with SCI (3 females and 9 males) were as follows (values expressed as mean \pm standard deviation): age $35.1 \pm 11.9 \mathrm{yr}$, body mass index $25.8 \pm 4.9 \mathrm{~kg} / \mathrm{m}^{2}$, and duration of injury $6.7 \pm 5.9 \mathrm{yr}$. The SCI group included five people with incomplete tetraplegia (ASIA C or D) and seven people with paraplegia (4 complete [ASIA A] and 3 incomplete [ASIA C or D]). Participants with SCI were grouped into the $\mathrm{C} 4-\mathrm{T} 5$ and $\mathrm{T} 6-\mathrm{T} 12$ groups to examine the effect of injury level on HRV and sacral skin perfusion. The C4-T5 group included seven people (1 complete and 6 incomplete), and the T6-T12 group included five people (3 complete and 2 incomplete). Three participants took baclofen for muscle spasticity, one took levothyroxine for hypothyroidism, one took sulfamethoxazole and trimethoprim for urinary tract infection, and one took tolterodine for overactive bladder. No participant experienced symptoms of autonomic dysreflexia during the experiment. Participants in the nondisabled control group did not have any diagnosed cardiovascular, metabolic, or neurological diseases. The demographic data of the nondisabled control group were as follows: age $26.1 \pm 5.8 \mathrm{yr}$, body mass index $23.5 \pm$ $2.8 \mathrm{~kg} / \mathrm{m}^{2}$, and seven females and seven males.

\section{Instrumentation}

A three-lead electrocardiographic monitor (ECG100C, Biopac Systems; Goleta, California) was used to record heart rate signal. The sampling rate was $1,000 \mathrm{~Hz}$ and the bandpass filter of $0.5-32 \mathrm{~Hz}$ was used to record the heart rate signal. AcqKnowledge software (Biopac Systems) was used to quantify R-R intervals. Laser Doppler flowmetry (LDF) (Periflux 5010, Perimed; Jarfalla, Sweden) was used to measure skin perfusion. LDF provides noninvasive measurement of skin perfusion at a depth of about $1 \mathrm{~mm}$.

\section{Procedures}

Posturally induced vasoconstriction was used to examine the relationship between the sympathovagal balance and skin perfusion in this study [26-27]. An increase in peripheral vascular resistance has been observed in the upright posture when compared with the supine posture. The responsible mechanism is an increased sympathovagal balance, which can be assessed by the LF to HF ratio of HRV. Room temperature was maintained at about $23^{\circ} \mathrm{C}$. Participants were kept in the laboratory for at least $30 \mathrm{~min}$ to acclimate to the room temperature. The skin sites for taping the probe and electrode were cleaned with alcohol pads. The LDF probe was taped on the skin over the sacrum (midpoint between right posterior superior iliac spine and spinal process) while the participant sat in the wheelchair. The electrocardiography (ECG) electrodes were placed on the right ventral wrist, right medial ankle, and left medial ankle according to the instructions of the Biopac ECG monitor. Sacral perfusion and ECG data were recorded in the upright posture for $10 \mathrm{~min}$. The participant placed arms on the armrest of the wheelchair and leaned slightly forward to avoid contact between the sacrum and the wheelchair seatback. The participant was then transferred to the mat table for another 10 min recording while he or she assumed a prone posture [13]. During the transfer, skin perfusion and heart rate signal were continuously recorded. Nondisabled controls followed the same procedures but changed from the upright posture to the prone posture without assistance.

\section{Data Analysis}

Skin perfusion measurements were averaged over a 10 min period in the upright and prone postures. Spectral analysis (Fourier transform with Hanning window) was used to calculate HRV parameters of the 10 min R-R intervals. MATLAB software (MATLAB 2009b, MathWorks; Natick, Massachusetts) was used to perform calculations. The definition of each HRV parameter used in this study was according to the published guideline [21]. HRV parameters analyzed in this study were LF (milliseconds squared, 0.04-0.15 Hz), HF (milliseconds squared, 0.15-0.4 Hz), LF (\%), HF (\%), LF to HF ratio, SDNN (milliseconds), pNN50 (\%), and rMSSD (milliseconds) [21]. LF (\%) was defined as power within LF divided by the total power of HRV minus power in very low frequency $(<0.04 \mathrm{~Hz})$. The Wilcoxon signed-ranked test was used to examine the differences of HRV and skin 
perfusion between upright and prone postures (withinsubjects test). The Mann-Whitney $U$ test was used to examine the difference in HRV and skin perfusion between people with SCI and nondisabled controls (between-subjects test). Relationships between sympathovagal balance and skin perfusion were assessed by Spearman correlation coefficients. For examining the influences of completeness and level of injury on HRV and skin perfusion, data were compared using one-tail Mann-Whitney U tests. All statistical tests were analyzed using SPSS 16 (IBM; Armonk, New York) and were performed at an alpha level of 0.05 .

\section{RESULTS}

Figure 1 shows an example of the time-series changes of skin perfusion (Figure 1(a) and (b)), R-R intervals (Figure 1(c) and (d)), and spectrum of HRV (Figure 1(e) and (f)) in response to postural changes in a nondisabled control (Figure 1(a), (c), and (e)) and a person with SCI (Figure 1(b), (d), and (f)). After the change from the upright to prone posture, skin perfusion showed an increase in the prone posture of the nondisabled control (Figure 1(a)), but not in the person with SCI (Figure 1(b)). R-R intervals increased in the nondisabled control (Figure 1(c)), but not in the person with SCI (Figure 1(d)). Spectral analysis revealed a decrease in the power of the LF of HRV and an increase in the power of the HF of HRV in the prone posture in the nondisabled control (Figure 1(e)), but not in the person with SCI (Figure 1(f)).

Figure 2(a) shows that the ratio of LF to total power was significantly higher in the upright posture than in the prone posture in nondisabled controls $(p<0.05)$, and the ratio of HF to total power was significantly lower in the upright posture than in the prone posture in nondisabled controls $(p<0.05)$. Figure 2(b) shows that the ratios of LF and HF to total power were not statistically different between the upright and prone postures in people with SCI (not significant [NS]).

The LF to HF ratio became larger in the upright posture when compared with the prone posture in nondisabled controls $(p<0.05)$, but not in people with SCI (NS) (Figure 3). The LF to HF ratio during the upright posture in nondisabled controls was significantly higher than the ratio during the upright posture in people with SCI $(p<$ $0.05)$. The LF to HF ratio during the prone posture in
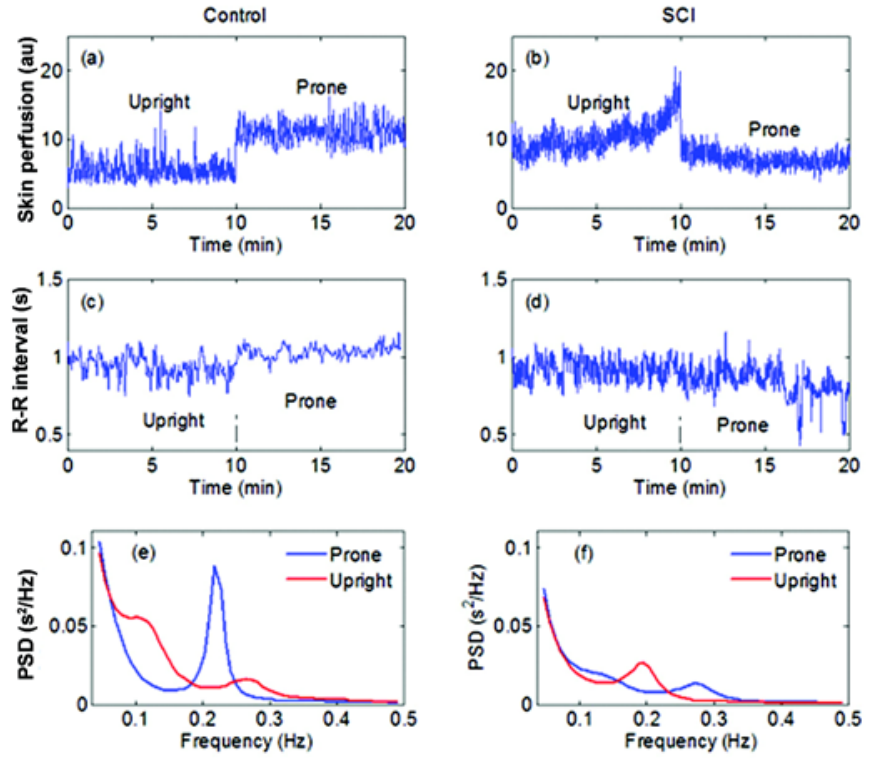

Figure 1.

Example of changes of skin perfusion, beat-to-beat interval of heart rate (R-R), and heart rate variability (HRV) during postural changes. Skin perfusion shows increase in prone posture in (a) nondisabled control, but not in (b) person with SCI. R-R intervals show increase in (c) nondisabled control, but not in (d) person with SCl. Spectral analysis reveals increase in power of low frequency of HRV in prone posture in (e) nondisabled control, but not in (f) person with SCI. PSD = power spectrum density.

nondisabled controls was lower than the ratio during the prone posture in people with SCI but was not statistically different (Figure 3).

Sacral skin perfusion was lower in the upright posture when compared with the prone posture in nondisabled controls $(p<0.05)$, while perfusion was not significantly different between the upright and prone postures in people with SCI (NS) (Figure 4). However, people with SCI showed an increasing trend in the prone posture (Figure 4).

The scatter plots of skin perfusion and sympathovagal balance during the upright and prone postures in nondisabled controls and people with SCI are shown in Figure 5. In nondisabled controls, lower skin perfusion and higher LF to HF ratios were observed in the upright posture ( $r=-0.54, p<0.05$ ), and higher skin perfusion and lower LF to HF ratios were observed in the prone posture $(r=0.71, p<0.05)$ (Figure 5(a)). In people with SCI, there were no obvious changes in skin perfusion and 

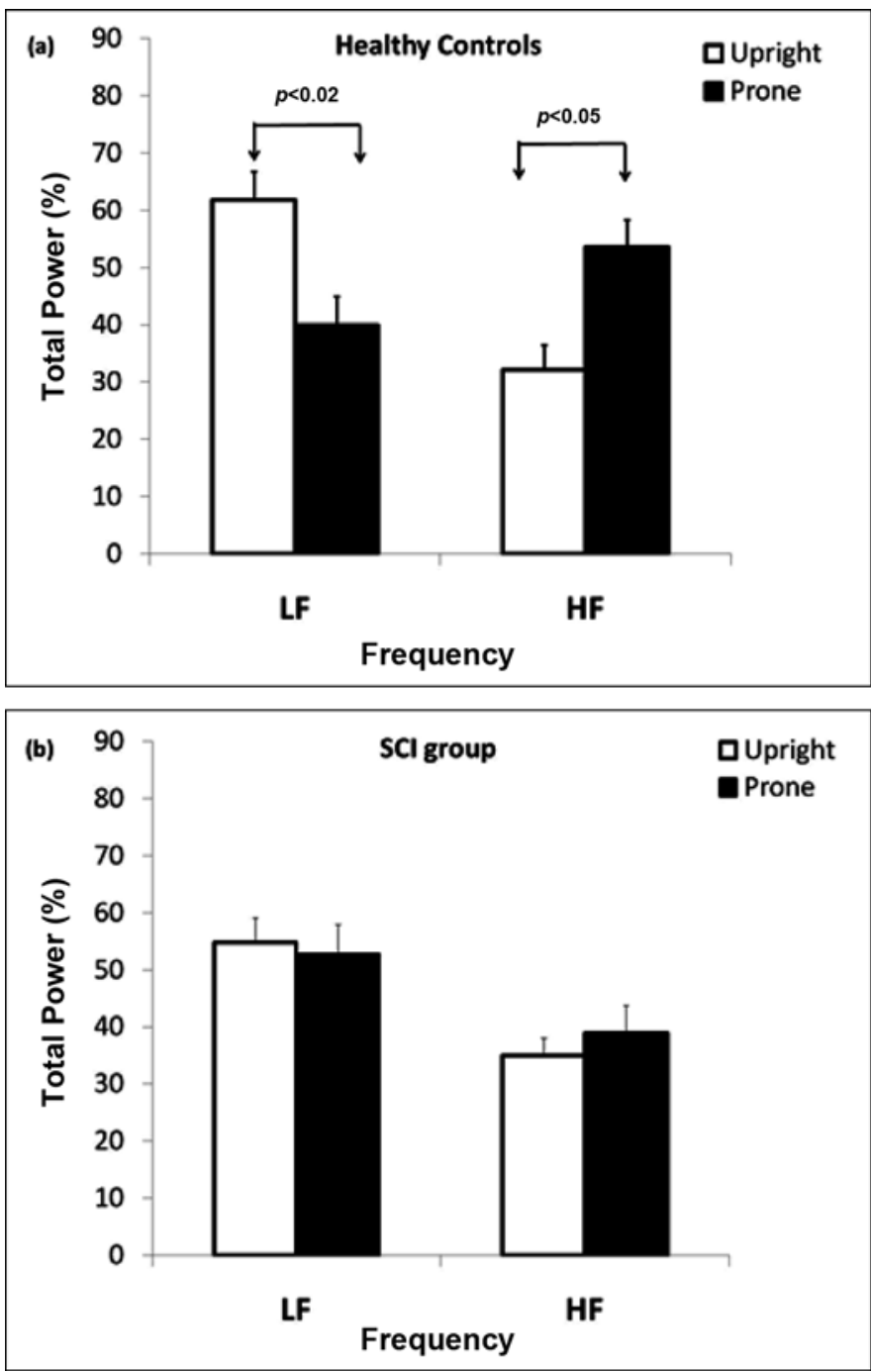

Figure 2.

Ratio of low frequency (LF) and high frequency (HF) to total power during upright and prone postures in (a) nondisabled controls and (b) people with spinal cord injury (SCl). (a) In nondisabled controls, LF is significantly higher in upright posture as compared with prone posture $(p<0.02)$ and HF is significantly lower in upright posture $(p<0.05)$. (b) There was no significant difference in LF and HF between upright and prone postures in people with $\mathrm{SCl}$ (not significant).

LF to HF ratios when changing from upright $(r=0.03$, NS) to prone ( $r=0.31$, NS) postures (Figure 5(b)). Very diverse responses of skin perfusion and LF to HF ratios were observed in people with SCI (Figure 5(b)).

The time domain HRV parameters are shown in Table 1. The parameters included mean R-R intervals, standard deviation of R-R intervals (SDNN), pNN50, and

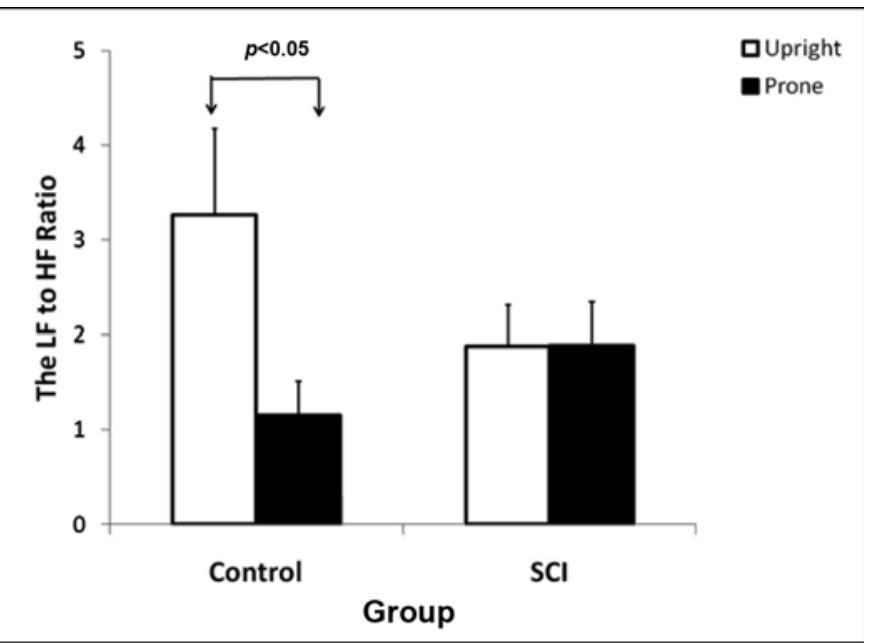

\section{Figure 3.}

Comparison of low frequency (LF) to high frequency (HF) ratio between upright and prone postures in nondisabled controls and people with spinal cord injury (SCl). Nondisabled controls have significantly higher ratio in upright posture as compared with prone posture $(p<0.05)$. LF to HF ratio does not show significant change between upright posture and prone posture in people with $\mathrm{SCl}$.

rMSSD. In people with SCI, no statistical differences were noted in time domain parameters between the upright and prone postures (NS). In nondisabled controls, mean R-R intervals and SDNN did not show significant differences between the upright and prone postures (NS), and pNN50 and rMSSD showed significant differences between the upright and prone postures $(p<0.05)$.

Comparison of the LF to HF ratio and skin perfusion in response to postural changes between the C4-T5 and T6-T12 groups did not show a significant difference (Table 2). However, the C4-T5 group showed a larger increase in skin perfusion in the prone posture when compared with the T6-T12 group ( $p=0.06)$. Comparison of the LF to HF ratio and skin perfusion in response to postural changes between the complete and incomplete groups did not show significant difference except a larger increase in skin perfusion in the incomplete injury group in the prone posture $(p<0.05)$ (Table 2).

\section{DISCUSSION}

We have demonstrated for the first time that the sympathovagal balance assessed by HRV was correlated 


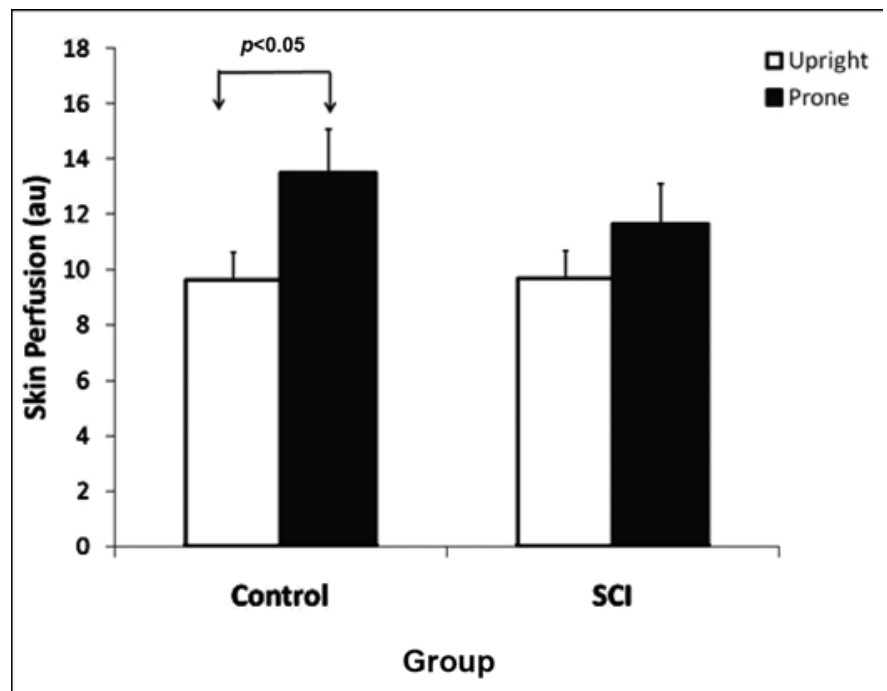

Figure 4.

Skin perfusion in upright and prone postures in people with spinal cord injury (SCl) and nondisabled controls. Nondisabled controls showed significant increase in prone posture as compared with upright posture $(p<0.05)$. People with $\mathrm{SCl}$ did not show significant increase.

with the skin vasoconstrictive response to postural changes. In nondisabled controls, the sympathovagal balance increased in the upright posture and decreased in the prone posture, and skin perfusion showed consistent changes with the degree of sympathovagal balance: lower perfusion as sympathovagal balance increased and higher perfusion as sympathovagal balance decreased. We further demonstrated that people with SCI had smaller changes in sympathovagal balance and in skin perfusion in response to postural changes as compared with nondisabled controls. Our findings support our hypothesis that impaired skin perfusion response to postural changes is associated with regulation of the sympathovagal balance following SCI. These results may provide initial evidence of the relationship between the sympathovagal balance assessed by HRV and skin perfusion. Our approach may be used to investigate the effects of SCI-induced autonomic damage on microvascular dysfunction.

Skin microcirculation has been widely used to evaluate effectiveness of preventive interventions or risk for pressure ulcers in people with SCI [13,28-29]. People with SCI have impaired microvascular reactivity in response to causative factors resulting from pressure ulcers [5]. Investigators have been trying to understand
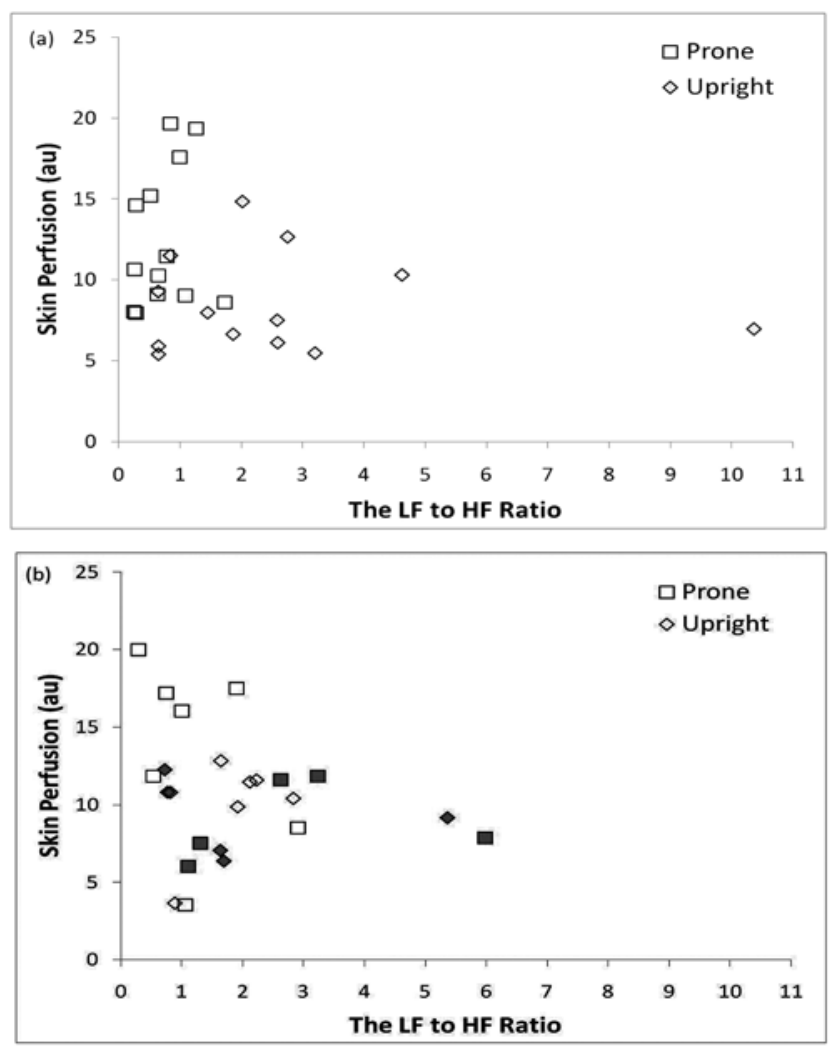

Figure 5.

Scatter plots of skin perfusion and sympathovagal balance during upright and prone postures in (a) nondisabled controls and (b) people with spinal cord injury (SCI). (a) In nondisabled controls, there is relationship showing that higher sympathovagal balance is associated with lower skin perfusion $(p<0.05)$. (b) In people with $\mathrm{SCl}$, relationship between sympathovagal balance and skin perfusion is not obvious (not significant). People with $\mathrm{SCl}$ between cervical 4 and thoracic (T)5 are expressed in white color and people with $\mathrm{SCl}$ between $\mathrm{T} 6$ and $\mathrm{T} 12$ are expressed in black color. HF = high frequency, LF = low frequency.

the effect of the completeness and level of SCI on microvascular dysfunction and to identify people at highest risk for pressure ulcers. However, there is no standard to document the influences of autonomic control on skin microcirculation in people with SCI. In order to solve this significant clinical problem, we assessed the response of HRV and skin perfusion in people with and without SCI. Thus, we examined whether the sympathovagal balance assessed by HRV could predict if postural changes affect skin perfusion. Based on our results, we demonstrated a potential to quantify the severity of autonomic damage on 
Table 1.

Time domain parameters of heart rate variability in nondisabled controls and people with spinal cord injury (SCI).

\begin{tabular}{|c|c|c|c|c|}
\hline Position & Mean R-R (ms) & SDNN (ms) & pNN50 (\%) & rMSSD (ms) \\
\hline \multicolumn{5}{|l|}{ Nondisabled Controls } \\
\hline Upright & $799.5 \pm 38.1$ & $92.2 \pm 11.7$ & $24.4 \pm 4.4$ & $57.8 \pm 10.6$ \\
\hline Prone & $910.5 \pm 41.2$ & $98.8 \pm 10.2$ & $42.9 \pm 5.8$ & $89.3 \pm 15.7$ \\
\hline \multicolumn{5}{|l|}{ SCI } \\
\hline Upright & $749.6 \pm 26.7$ & $87.1 \pm 21.1$ & $15.3 \pm 4.6$ & $60.9 \pm 18.2$ \\
\hline Prone & $802.6 \pm 22.4$ & $89.1 \pm 21.2$ & $16.6 \pm 4.9$ & $59.6 \pm 18.8$ \\
\hline
\end{tabular}

Note: Values expressed as mean \pm standard error of mean.

pNN50 = percentage of adjacent cycles that are $>50 \mathrm{~ms}$ apart, rMSSD = root-mean-square successive differences in milliseconds, R-R = beat-to-beat interval of heart rate, SDNN = standard deviation of all normal-to-normal intervals.

Table 2.

Heart rate variability and skin perfusion data in people with spinal cord injury ( $n=12)$ grouped into cervical (C)4-thoracic (T)5 and T6-T12 groups and complete and incomplete injury groups.

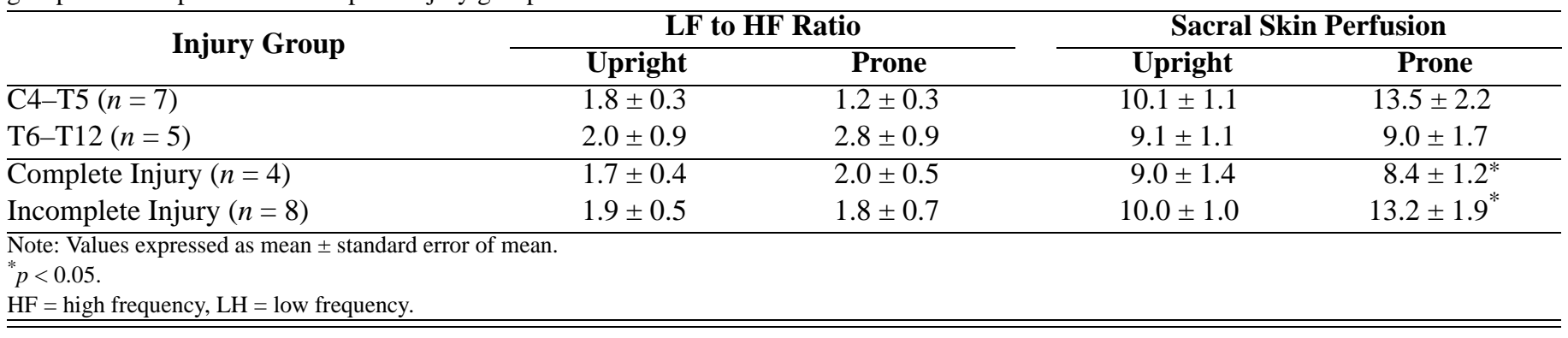

microvascular dysfunction in people with SCI. This means that as an injury becomes more severe, there should be less of a relationship between HRV and skin blood flow. This is also important for understanding the underlying pathologies causing microvascular dysfunction following SCI. For example, microvascular dysfunction may be caused by prolonged physical inactivity or impaired sympathovagal regulation [5]. The method used in this study might be used to differentiate physical inactivityinduced microvascular dysfunction [11,30]. Also, when comparing microvascular functions and responses, it is critical to understand the posture of the participant because of a potential influence on autonomic regulation. If skin perfusion is assessed during the upright posture, people with SCI may show larger microvascular impairments. This issue is important with respect to determining the effectiveness of wheelchair seat cushions and hospital mattresses. Future research needs to examine whether the testing posture of people with SCI affects skin perfusion in response to pressure loading or other causative factors of pressure ulcers.

We demonstrated the influences of sympathovagal balance assessed by HRV on skin perfusion in this study. The finding does not conflict with our previous studies using wavelet analysis to quantify activities of physiological control mechanisms from skin blood flow oscillations [7,3132]. Our previous studies have shown that five characteristic frequencies embedded in skin BFOs are associated with metabolic, neurogenic, myogenic, respiratory, and cardiac origins. The evidence that $0.02-0.05 \mathrm{~Hz}$ is from neurogenic origins seems to be supported by Söderström et al. [8] and Wilson et al. [33], although some controversy still exists. In order to further examine the role of the sympathovagal system on skin perfusion following SCI, we quantified HRV parameters to show a relationship between the sympathovagal balance (the LF to HF ratio) and skin perfusion. Based on the current study, we will be able to investigate the relationship between the sympathovagal balance assessed by HRV and the neurogenic control assessed by BFO. Through these studies, we intend to develop objective tools to quantify the effects of autonomic damage on microvascular dysfunction in people with SCI. With an improved assessment, clinicians will be able to predict autonomic-related diseases and evaluate the effectiveness of preventive interventions on enhancing microvascular function following SCI. 
Our findings support the use of the LF to HF ratio to assess the sympathovagal balance. Our results showed that the LF to HF ratio (sympathovagal balance) significantly changed as the nondisabled controls moved from the upright to prone postures. In order to validate whether the LF to HF ratio can be used to quantify the sympathovagal balance in people with SCI, several studies have investigated changes of LF to $\mathrm{HF}$ ratios in response to various stimuli [2,16-19]. These studies contain conflicting results regarding whether the LF to HF ratio can be used to assess the severity of autonomic dysfunction following SCI. More research is recommended to clarify this issue [2]. Our study supports the opinion that HRV can be used to assess sympathovagal dysfunction in people with SCI. The inconsistency of HRV results in the literature may have occurred because of existing cardiovascular diseases in the participants with SCI $[2,24]$. In our study, people with pre-existing cardiovascular diseases were excluded to create a more homogeneous response in people with SCI as compared with nondisabled controls. Future studies need to develop new methods of analysis to assess the sympathovagal balance in people with SCI with and without cardiovascular diseases.

Our data showed that people with SCI had an LF to HF ratio of about 1.9 in both upright and prone postures. The value was higher than the ratio (1.1) in the prone posture and lower than the ratio (3.3) in the upright posture of the nondisabled controls. These results are consistent with previous studies that examined sympathovagal functions in people with chronic SCI [34]. In people with cervical and high thoracic SCI, the sympathetic system loses its innervation with the cardiovascular system while the parasympathetic system (vagal nerve) remains intact [4]. As a consequence immediately following SCI, people with SCI have a decrease of sympathovagal balance. As time proceeds, people with chronic SCI show a better sympathovagal balance potentially because of decreased control of the vagal nerve [4]. By assessing changes of the LF to HF ratio, clinicians might monitor the recovery of sympathovagal regulations of skin blood flow. To explore the effect of the level and completeness of SCI on autonomic dysfunction and its influences on microvascular reactivity, we compared the HRV and skin perfusion in the C4-T5 and T6-T12 groups and the complete and incomplete injury groups. Our results showed that the C4-T5 group has a larger increase in skin perfusion in the prone posture when compared with the T6-T12 group. This seems contradictory to our understanding, i.e., people with higher injury level (T6 and above) will lose sympathetic outflow to the heart, resulting in an impaired microvascular function. This could be explained by the completeness of injury in two groups. The C4-T5 group mainly consisted of incomplete injury, while the T6-T12 group mainly consisted of complete injury. This statement was also supported by our results that compared HRV and skin perfusion in the complete and incomplete groups. The results showed that people with incomplete injury have a significant increase in skin perfusion in response to postural changes as compared with people with complete injury. To better understand the microvascular dysfunction in people with SCI, future research needs to examine the effect of level and completeness of SCI on the sympathetic outflow to the heart and skin.

There were limitations to this study. First, we did not control or measure the respiration rate of research participants during the experiments. This limited our ability to analyze the effects of respiration rate on the changes of power of HF of HRV [24]. However, in this study, we also calculated rMSSD and pNN50 indices to assess the parasympathetic activity and found their values were consistent with changes of power of LF and HF of HRV. Future studies should control or record the respiration rate to minimize its influences on HRV values. Second, we only recruited 12 people with SCI into this study. Since this was a proof of concept study, we did not intend to recruit a large number of participants with SCI. Our results confirmed our hypothesis that the relationship between the sympathovagal balance and skin perfusion may be used to quantify the effects of SCI-induced autonomic impairment on microvascular dysfunction. More studies are required to determine the influences of injury level and completeness on the changes of HRV and skin perfusion in response to postural changes. Third, the SCI group had more male participants and was older compared with the control group. The age and sex issue may affect the validity of our results. However, the age and sex effect is a minor confounding variable when compared with our intervention, postural changes [35-36]. Thus, the difference of HRV and skin perfusions between people with SCI and nondisabled controls are less likely because of an unequal distribution of ages and sexes. Further studies should consider using a sex-matched research design. Last, the LF of HRV may reflect baroreflex activity rather than sympathovagal activity [23]. 


\section{CONCLUSIONS}

Although the specific pathophysiological meaning of HRV in people with SCI remains to be determined, the use of HRV seems promising for assessing the influences at various levels of SCI on microvascular dysfunction. In this study, our results showed that the LF to HF ratio was a sensitive index to evaluate the changes of sympathovagal balance in response to postural changes. The relationship between HRV and skin blood flow appears to be affected and diminished by SCI. Whether this departure from what is normal can be used as a clinical indicator remains to be determined.

\section{ACKNOWLEDGMENTS}

\author{
Author Contributions: \\ Study concept and design: Y. K. Jan. \\ Acquisition of data: Y. K. Jan, M. Anderson. \\ Analysis and interpretation of data: Y. K. Jan, M. Anderson, \\ J. Soltani, S. Burns, R. D. Foreman. \\ Drafting of manuscript: Y.K. Jan. \\ Critical revision of manuscript for important intellectual content: \\ R. D. Foreman. \\ Statistical analysis: Y. K. Jan. \\ Obtained funding: Y. K. Jan. \\ Administrative, technical, or material support: Y. K. Jan. \\ Study supervision: Y. K. Jan.
}

Financial Disclosures: The authors have declared that no competing interests exist.

Funding/Support: This material was based on work supported by the National Institutes of Health (grant P20GM103447) and the Christopher and Dana Reeve Foundation (grant JA2-0701-2).

Additional Contributions: The authors would like to thank Katrina Shaklee at the Wellness Center at the University of Central Oklahoma for assistance recruiting people with SCI. Dr. Jan is now with the Rehabilitation Research Laboratory, Department of Kinesiology and Community Health, University of Illinois at Urbana-Champaign.

Institutional Review: We received human subjects approval from the University of Oklahoma Health Sciences Center, Oklahoma City, Oklahoma. All participants gave informed consent.

Participant Follow-Up: A copy of this article will be mailed to each study participant.

\section{REFERENCES}

1. Claydon VE, Krassioukov AV. Orthostatic hypotension and autonomic pathways after spinal cord injury. J Neurotrauma. 2006;23(12):1713-25. [PMID:17184183] http://dx.doi.org/10.1089/neu.2006.23.1713
2. Claydon VE, Krassioukov AV. Clinical correlates of frequency analyses of cardiovascular control after spinal cord injury. Am J Physiol Heart Circ Physiol. 2008;294(2): H668-78. [PMID:18024546] http://dx.doi.org/10.1152/ajpheart.00869.2007

3. Alexander MS, Biering-Sorensen F, Bodner D, Brackett NL, Cardenas D, Charlifue S, Creasey G, Dietz V, Ditunno J, Donovan W, Elliott SL, Estores I, Graves DE, Green B, Gousse A, Jackson AB, Kennelly M, Karlsson AK, Krassioukov A, Krogh K, Linsenmeyer T, Marino R, Mathias CJ, Perkash I, Sheel AW, Schilero G, Schurch B, Sonksen J, Stiens S, Wecht J, Wuermser LA, Wyndaele JJ. International standards to document remaining autonomic function after spinal cord injury. Spinal Cord. 2009;47(1):36-43.

[PMID:18957962]

http://dx.doi.org/10.1038/sc.2008.121

4. Krassioukov AV, Karlsson AK, Wecht JM, Wuermser LA, Mathias CJ, Marino RJ; Joint Committee of American Spinal Injury Association and International Spinal Cord Society. Assessment of autonomic dysfunction following spinal cord injury: rationale for additions to International Standards for Neurological Assessment. J Rehabil Res Dev. 2007;44(1):103-12. [PMID:17551864] http://dx.doi.org/10.1682/JRRD.2005.10.0159

5. Jan YK, Brienza D. Tissue mechanics and blood flow factors in pressure ulcers of people with spinal cord injury. In: Gefen A, editor. The pathomechanics of tissue injury and disease, and the mechanophysiology of healing. Kerala (India): Research Signpost; 2009. p. 241-59.

6. Stefanovska A, Bracic M, Kvernmo HD. Wavelet analysis of oscillations in the peripheral blood circulation measured by laser Doppler technique. IEEE Trans Biomed Eng. 1999;46(10):1230-39. [PMID:10513128]

http://dx.doi.org/10.1109/10.790500

7. Jan YK, Struck BD, Foreman RD, Robinson C. Wavelet analysis of sacral skin blood flow oscillations to assess soft tissue viability in older adults. Microvasc Res. 2009;78(2):162-68. [PMID:19465031] http://dx.doi.org/10.1016/j.mvr.2009.05.004

8. Söderström T, Stefanovska A, Veber M, Svensson H. Involvement of sympathetic nerve activity in skin blood flow oscillations in humans. Am J Physiol Heart Circ Physiol. 2003;284(5):H1638-46. [PMID:12679328]

9. Landsverk SA, Kvandal P, Bernjak A, Stefanovska A, Kirkeboen KA. The effects of general anesthesia on human skin microcirculation evaluated by wavelet transform. Anesth Analg. 2007;105(4):1012-19. [PMID:17898381] http://dx.doi.org/10.1213/01.ane.0000281932.09660.96

10. Previnaire JG, Soler JM, El Masri W, Denys P. Assessment of the sympathetic level of lesion in patients with spinal cord injury. Spinal Cord. 2009;47(2):122-27. 
JRRD, Volume 50, Number 2, 2013

\section{[PMID:18663374]}

http://dx.doi.org/10.1038/sc.2008.87

11. Jan YK, Brienza DM, Geyer MJ. Analysis of week-toweek variability in skin blood flow measurements using wavelet transforms. Clin Physiol Funct Imaging. 2005; 25(5):253-62. [PMID:16117727] http://dx.doi.org/10.1111/j.1475-097X.2005.00621.x

12. Nicotra A, Young TM, Asahina M, Mathias CJ. The effect of different physiological stimuli on skin vasomotor reflexes above and below the lesion in human chronic spinal cord injury. Neurorehabil Neural Repair. 2005;19(4): 325-31. [PMID:16263964] http://dx.doi.org/10.1177/1545968305281210

13. Jan YK, Brienza DM, Boninger ML, Brenes G. Comparison of skin perfusion response with alternating and constant pressures in people with spinal cord injury. Spinal Cord. 2011;49(1):136-41. [PMID:20514054] http://dx.doi.org/10.1038/sc.2010.58

14. Olesen CG, de Zee M, Rasmussen J. Missing links in pressure ulcer research-an interdisciplinary overview. J Appl Physiol. 2010;108(6):1458-64. [PMID:20299616] http://dx.doi.org/10.1152/japplphysiol.01006.2009

15. Weimer LH. Autonomic testing: common techniques and clinical applications. Neurologist. 2010;16(4):215-22. [PMID:20592565] http://dx.doi.org/10.1097/NRL.0b013e3181cf86ab

16. La Fountaine MF, Wecht JM, Spungen AM, Bauman WA. Intra-inter visit reproducibility of short-term linear and nonlinear measurement of heart rate variability in tetraplegia and neurologically intact controls. Physiol Meas. 2010; 31(3):363-74. [PMID:20130343] http://dx.doi.org/10.1088/0967-3334/31/3/006

17. Ditor DS, Kamath MV, Macdonald MJ, Bugaresti J, McCartney N, Hicks AL. Reproducibility of heart rate variability and blood pressure variability in individuals with spinal cord injury. Clin Auton Res. 2005;15(6):387-93. [PMID:16362541] http://dx.doi.org/10.1007/s10286-005-0293-4

18. Millar PJ, Rakobowchuk M, Adams MM, Hicks AL, McCartney N, MacDonald MJ. Effects of short-term training on heart rate dynamics in individuals with spinal cord injury. Auton Neurosci. 2009;150(1-2):116-21. [PMID:19406691] http://dx.doi.org/10.1016/j.autneu.2009.03.012

19. Claydon VE, Steeves JD, Krassioukov A. Orthostatic hypotension following spinal cord injury: understanding clinical pathophysiology. Spinal Cord. 2006;44(6):341-51. [PMID:16304564] http://dx.doi.org/10.1038/sj.sc.3101855

20. Tsuji H, Larson MG, Venditti FJ Jr, Manders ES, Evans JC, Feldman CL, Levy D. Impact of reduced heart rate variability on risk for cardiac events. The Framingham Heart
Study. Circulation. 1996;94(11):2850-55.

[PMID:8941112]

http://dx.doi.org/10.1161/01.CIR.94.11.2850

21. Task Force of the European Society of Cardiology and the North American Society of Pacing and Electrophysiology. Heart rate variability: standards of measurement, physiological interpretation and clinical use. Circulation. 1996; 93(5):1043-65. [PMID:8598068] http://dx.doi.org/10.1161/01.CIR.93.5.1043

22. Akselrod S. Spectral analysis of fluctuations in cardiovascular parameters: a quantitative tool for the investigation of autonomic control. Trends Pharmacol Sci. 1988;9(1):6-9. [PMID:3245076] http://dx.doi.org/10.1016/0165-6147(88)90230-1

23. Moak JP, Goldstein DS, Eldadah BA, Saleem A, Holmes C, Pechnik S, Sharabi Y. Supine low-frequency power of heart rate variability reflects baroreflex function, not cardiac sympathetic innervation. Heart Rhythm. 2007;4(12): 1523-29. [PMID:17997358] http://dx.doi.org/10.1016/j.hrthm.2007.07.019

24. Stein PK, Kleiger RE. Insights from the study of heart rate variability. Annu Rev Med. 1999;50:249-61.

[PMID:10073276]

http://dx.doi.org/10.1146/annurev.med.50.1.249

25. Bigger JT Jr, Fleiss JL, Steinman RC, Rolnitzky LM, Kleiger RE, Rottman JN. Correlations among time and frequency domain measures of heart period variability two weeks after acute myocardial infarction. Am J Cardiol. 1992;69(9):891-98. [PMID:1550018] http://dx.doi.org/10.1016/0002-9149(92)90788-Z

26. Jepsen H, Gaehtgens P. Postural vascular response vs. sympathetic vasoconstriction in human skin during orthostasis. Am J Physiol. 1995;269(1 Pt 2):H53-61. [PMID:7631874]

27. Mayrovitz HN. Posturally induced leg vasoconstrictive responses: relationship to standing duration, impedance and volume changes. Clin Physiol. 1998;18(4):311-19. [PMID:9715756] http://dx.doi.org/10.1046/j.1365-2281.1998.00099.x

28. Bogie KM, Nuseibeh I, Bader DL. Early progressive changes in tissue viability in the seated spinal cord injured subject. Paraplegia. 1995;33(3):141-47. [PMID:7784116] http://dx.doi.org/10.1038/sc.1995.31

29. Sae-Sia W, Wipke-Tevis DD, Williams DA. The effect of clinically relevant pressure duration on sacral skin blood flow and temperature in patients after acute spinal cord injury. Arch Phys Med Rehabil. 2007;88(12):1673-80. [PMID:18047884] http://dx.doi.org/10.1016/j.apmr.2007.07.037

30. Beckers F, Verheyden B, Aubert AE. Aging and nonlinear heart rate control in a healthy population. Am J Physiol Heart Circ Physiol. 2006;290(6):H2560-70. 


\section{[PMID:16373585]}

http://dx.doi.org/10.1152/ajpheart.00903.2005

31. Geyer MJ, Jan YK, Brienza DM, Boninger ML. Using wavelet analysis to characterize the thermoregulatory mechanisms of sacral skin blood flow. J Rehabil Res Dev. 2004;41(6A):797-806. [PMID:15685468] http://dx.doi.org/10.1682/JRRD.2003.10.0159

32. Jan YK, Brienza DM, Geyer MJ, Karg P. Wavelet-based spectrum analysis of sacral skin blood flow response to alternating pressure. Arch Phys Med Rehabil. 2008; 89(1):137-45. [PMID:18164343] http://dx.doi.org/10.1016/j.apmr.2007.07.046

33. Wilson TE, Zhang R, Levine BD, Crandall CG. Dynamic autoregulation of cutaneous circulation: differential control in glabrous versus nonglabrous skin. Am J Physiol Heart Circ Physiol. 2005;289(1):H385-91. [PMID:15749747] http://dx.doi.org/10.1152/ajpheart.00622.2004

34. Grimm DR, De Meersman RE, Almenoff PL, Spungen AM, Bauman WA. Sympathovagal balance of the heart in subjects with spinal cord injury. Am J Physiol. 1997;272(2 Pt 2):H835-42. [PMID:9124446]

35. Bircher A, de Boer EM, Agner T, Wahlberg JE, Serup J. Guidelines for measurement of cutaneous blood flow by laser Doppler flowmetry. A report from the Standardization
Group of the European Society of Contact Dermatitis. Contact Dermatitis. 1994;30(2):65-72. [PMID:8187504] http://dx.doi.org/10.1111/j.1600-0536.1994.tb00565.x

36. Wecht JM, Weir JP, Bauman WA. Blunted heart rate response to vagal withdrawal in persons with tetraplegia. Clin Auton Res. 2006;16(6):378-83. [PMID:17066256] http://dx.doi.org/10.1007/s10286-006-0367-y

Submitted for publication August 1, 2011. Accepted in revised form June 21, 2012.

This article and any supplementary material should be cited as follows:

Jan YK, Anderson M, Soltani J, Burns S, Foreman RD. Comparison of changes in heart rate variability and sacral skin perfusion in response to postural changes in people with spinal cord injury. J Rehabil Res Dev. 2013;50(2): 203-14.

http://dx.doi.org/10.1682/JRRD.2011.08.0138

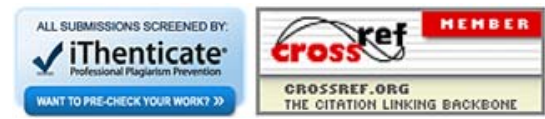


